\title{
THE DIFFERENCE BETWEEN THE EFFECT OF ELECTROMAGNETIC AND MAGNETIC FIELDS ON THE VISCOSITY COEFFICIENTS OF CUTTING FLUIDS USED IN CUTTING PROCESSES
}

\author{
Umidjon Mardonov ${ }^{1}$, Muhammad Turonov $^{1}$, Andrey Jeltukhin ${ }^{1}$, Yahyojon Meliboyev ${ }^{1}$ \\ ${ }^{1}$ Tashkent State Technical University \\ Email: red engineer94@mail.ru
}

\begin{abstract}
In this paper, authors investigated the difference between the influence of permanent magnetic field and pulsating electromagnetic field on cutting fluids. Two essential physical parameters of the cutting fluids, dynamic and kinematic viscosity coefficients, were measured during the experiment in the same laboratory condition. The results obtained from the experiments show that there is a noticeable difference between the impact of permanent magnetic field and pulsating electromagnetic field on cutting fluids. Three different types of fluids studied in the paper and these fluids are the most popular lubricating cooling fluids used in cutting process in manufacturing. All the experiments conducted in the same laboratory condition with the magnetic field strength of $40 \mathrm{mT}, 60 \mathrm{mT}$ and $80 \mathrm{mT}$.
\end{abstract}

Keywords: Cutting Fluid, Electromagnetic Field, Magnetic Field, Machining, Viscosity.

\section{Introduction}

It is known that the molecular structure of fluids is changed by the Magnetic field. Chang and Weng said that after magnetic water treatment, in molecule clusters of water, the volume of hydrogen bonds increased [1]. Another study shows that water surface tension was degraded and viscosity coefficient escalated after the effect of magnetized water treatment. Cho and Lee studied the influence of magnetic field on water surface tension [2]. They investigated that the surface tension of magnetized water did not significantly relate to the water hardness. They also found that because of the increased number of colloidal particles under the influence of the magnetic field, the surface tension of magnetized water decreased.

The experiment they conducted in dye-injection, they noticed that in not magnetized water, dye drops extend along the radial direction fast; or more clearly, not magnetized water was not penetrated by dye, however it was always on the water surface. However, when they magnetized the water, a lot of dye drops left the surface of the magnetized water. Famous scientists Amiri and Dadkhah [3] were obtained some controversial results, they studied the difference between the surface tension of magnetized and not magnetized water. They conducted about 200 tests in six-month, and they found that there was noticeable decrease on the surface tension of magnetized water it was because of magnetic field treatment. Zhian Liu [4] investigated that the low-frequency magnetic field made by permanent magnets is an oscillating magnetic field and it emerges a strong shock wave.

As a result of that low-frequency oscillating magnetic field, metabolism was delayed and microbial were died. Christian Baresel et al. found that the magnetic particles produced in their experiment worked perfectly for magnetic separation, even if extra optimizations were possible. They said that the probability to recuperate a main part of the particles scaled-up system could be good in the future [5]. Mardonov Umidjon et al. studied the influence of the stationary magnetic field on the physical and chemical properties of three different lubricating cooling liquids. They investigated that influence of the magnetic field on boiling point, density and kinematic viscosity coefficient of cutting fluids depends on magnetic field strengths.

Moreover, they found that magnetic treatment decreases the boiling point and kinematic viscosity coefficient of liquids and increases the density of liquids $[6-7,18]$.

However, this issue about magnetic field treatment of liquids remains controversial. The complete understanding of the influence of magnetic fields on fluids has a great impact on agriculture, industry, and other fields [8-10]. Some scientists are working on the issue of magnetic field treatment of water, but there lack researches on this issue, especially the magnetic field effect on different liquids. 


\section{Methods}

The first liquid (liquid-1) was tap water and it was from a local water supply company (Suvsoz, Uzbekistan), the second and the third liquids (liquid2 and liquid-3) were taken from a local manufacturing factory (Meridian-A). These three liquids are used as lubricating cooling liquids in the "Meridian-A" manufacturing factory. The second liquid, liquid-2 was a compound of water and $\mathrm{K}_{2} \mathrm{Cr}_{2} \mathrm{O}_{7}$ powder with $0.2 \%$ concentration, or more accurately we dissolved $m=50$ gram of $\mathrm{K}_{2} \mathrm{Cr}_{2} \mathrm{O}_{7}$ powder in $m_{0}=25 \mathrm{~kg}$ of water. The Lubricating cooling liquid with this concentration is used in machining details which materials are not hard alloys in lathes in the factory. The Liquid-3 is also a lubricating cooling fluid and it is mainly used in machining details, which the materials are hard alloys. It is a $5 \%$ concentration of BM-76M (cutting fluid) with water.

The magnetizing equipment UMD-1 (Fig. 1) was developed to take a permanent magnetic field for magnetizing flowing liquids, there are eight ceramic magnets with the size of $120 \times 80 \times 16$ (length, width, height), the minimum strength that can be taken from the magnets is $40 \mathrm{mT}$. The details of UMD-1 magnetizing equipment are given in Fig. 1

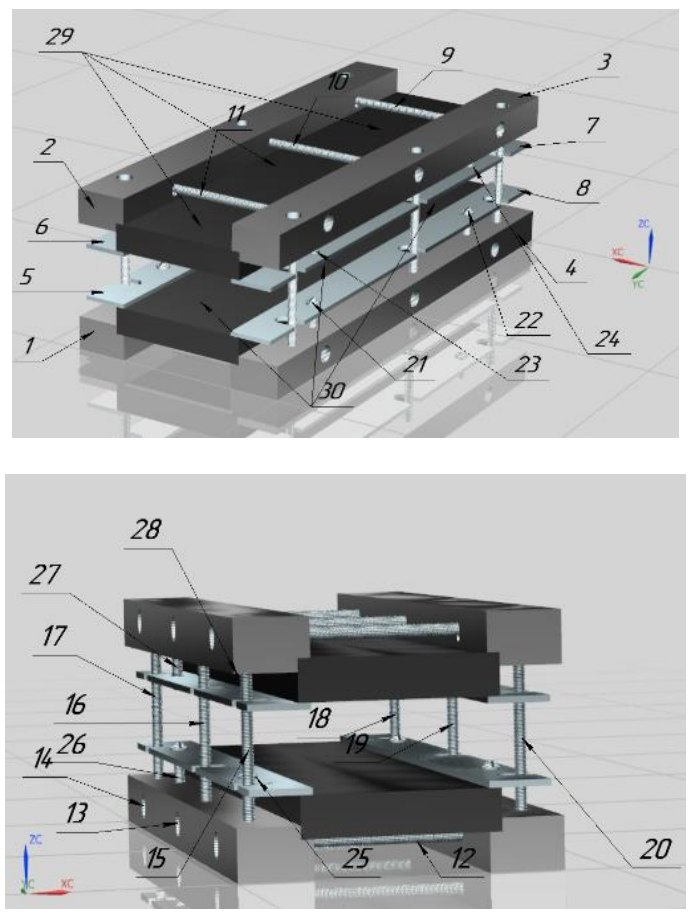

Figure 1: UMD-1 magnetizing device. 1-4 - Supports, 58 - magnet holders, 9-14 - Horizontal hexagon bolts, 15-20 - Vertical hexagon bolts, 21-28 - Screws to fix magnet holders, 29,30 - Magnets.
Fig. 1 shows that, magnets $(29,30)$ are placed on lower $(1,4)$ and upper $(2,3)$ supports with different poles. Long horizontal hexagon bolts $(9,10,11,12,13,14)$ are fixed according to width of the magnets, and holders $(5,6,7,8)$ are fixed to hold magnets hard by screw (21-28). Holders and supports are developed to change the distance between them. bottom and top supports have the same construction and the distance between the lower and upper supports can be changed easily by vertical hexagon bolts (15-20). Changing the distance between the bottom and top supports is purposed to change the distance between the magnets placed on lower and upper supports. We can easily change the magnetic field strength by changing the distance between lower and upper magnets. Moreover, the distance between the horizontal supports can be changed according to the sizes of magnets. The design of the UMD-1 magnetizing device is universal because it is possible to place magnets with different sizes (length, width, height). Magnets that have a minimum size of 20x10x5 (mm) and a maximum size of $300 \times 80 \times 30 \mathrm{~mm}$ can be placed on the UMD-1 magnetizing device. The distance can be changed from $0 \mathrm{~mm}$ to $50 \mathrm{~mm}$ with respect to the sizes of the magnets placed on the supports. Furthermore, the UMD-1 magnetizing device is very portable and it is very easy to change its position on cutting machines.

The Polyvinyl chloride pipe is places between the top and bottom magnets, and the diameter of the pipe is $10 \mathrm{~mm}$. The length of the magnetic field was $480 \mathrm{~mm}$ and the Polyvinyl chloride pipe passed through the magnetic fields. A fluid flow through the Polyvinyl chloride pipe turned into magnetized fluid. All of the samples circulated at $0.4 \mathrm{~m} / \mathrm{s}$ of flowing speed for 30 minutes through the UMD-1 magnetizing devise. The scheme of the magnetizing process is given in Fig. 2.

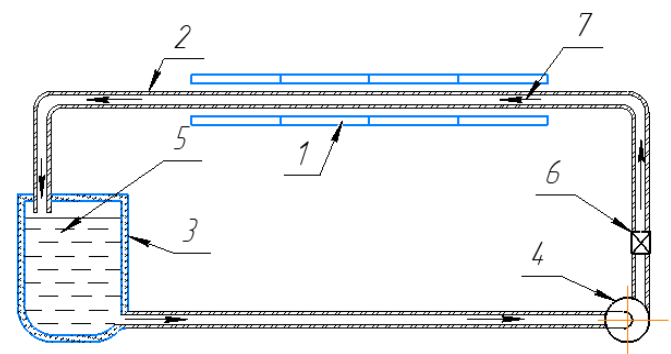

Figure 2: UMD-1 magnetizing equipment, 2 - PVC pipe, 3 - container, 4 - pump, 5 - liquid, 6 - tap, 7 - flowing direction of the liquid.

The SMD-2 special magnetizing laboratory device based on a solenoid was projected and built to make a pulsating electromagnetic field for magnetizing liquids (Fig. 3). 


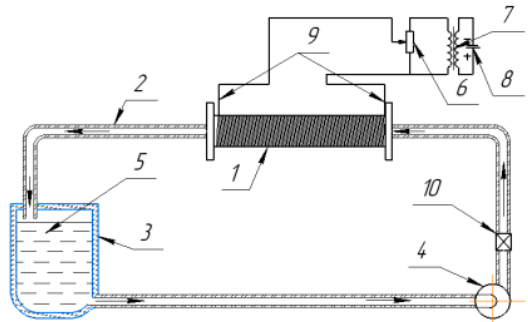

Figure 3: SMD-2 magnetizing device. 1-Solenoid, 2Ceramic pipe, 3-Container, 4-Pump, 5-Liquid, 6Rheostat, 7-Transformer, 8-Voltage, 9- Pulse signal.

Container 3 is filled with 8 litres of sampled liquid 5, and the liquid on the container is removed from the container using a pump 4 through ceramic tubes 2 . The liquid that moving through the pipe following the direction that is shown in the diagram passes through the centre of solenoid 1 with a diameter of $18.5 \mathrm{~mm}$ and is poured back into the container. The liquid coming through the tube passes through the centre of the solenoid, which creates a pulsating electromagnetic field, and then turns into a magnetized liquid and is poured back into the container. The pressure of the liquid is controlled by tap 10 and the required pressure is obtained. After a few minutes of the cycle, the liquid in the container becomes fully magnetized and the viscosity coefficient of the liquid in it is measured. The solenoid created for the experiment was made by wrapping an insulated copper wire, the wire has $1.06 \mathrm{~mm}$ of diameter in a plasma coil with a radius of $25 \mathrm{~mm}$, and the number of turns of wires in it was 2100. The $220 \mathrm{~V}$ voltage from the source (9) is reduced by transformer 7 and passed through a rheostat 6 in order to turn to a regulated pulsating voltage 9. The pulsating voltage from the diode bridge is transmitted to the solenoid and a pulsating electromagnetic field is created. The voltage value is controlled using a rheostat and is set to the desired voltage.

The magnetic field strength, magnetizing condition, and velocity of liquids were considered as three influential factors that affected the changes caused by the magnetic field $[11,12]$. Therefore, all of the experiments have been conducted in the same laboratory condition and the specification of the laboratory condition is given in table 1 . The laboratory was 440 meters high above sea level and atmospheric pressure was $\mathrm{P}=770 \mathrm{mmHg}$ when all of the experiments were conducted. The PVC pipe which diameter was $\mathrm{d}=10 \mathrm{~mm}$ was used for passing fluids through an electromagnetic field that was made using a solenoid.

The flowing velocity of liquid was $v=0.4 \mathrm{~m} / \mathrm{s}$ while the experiment was conducting.

\section{- Measurement of the dynamic viscosity}

There are a lot of types of viscometers which measures the dynamic viscosity coefficient.

The Stokes viscometer's theory is based on the study of the free fall of the ball in experimented fluid.
The Stokes viscometer is usually a crystal-clear cylinder filled with the studied fluid. The time $\Delta t$ of the falling ball between two lines at a distance $\mathrm{x}=\Delta \mathrm{L}$ is measured by special timer. The material of the ball can be chosen according to laboratory condition, it can be iron or any type of steel. Taking into account a simple formula for the final velocity $v \infty=\Delta \mathrm{L} / \Delta \mathrm{t}$ and paying attention to the equation [1] we finally reached an equation to find the dynamic viscosity coefficient.

$$
\mu=\frac{2}{9} g r^{2}\left(\rho_{\text {ball }}-\rho\right) \frac{\Delta t}{\Delta l}
$$

Where:

$\mathrm{g}$ - the free fall acceleration (gravity),

$\mathrm{r}$ - the ball radius,

Pball - the density of the ball material,

$\rho$ - the density of the studied fluid,

$\Delta t$ - the time of the free fall of the ball between the two marks placed at the distance $\Delta \mathrm{L}$.

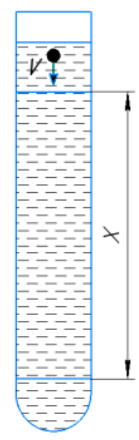

Figure 4: A device for measuring the dynamic viscosity coefficient of a fluid according to Stock's method

The diameter of the ball is measured to the accuracy of $0.01 \mathrm{~mm}$ using a micrometre. The ball is thrown into the liquid inside the cylinder (the ball should be thrown as close to the cylinder axis and the surface of the fluid as possible). The stopwatch measures the time which the ball passes through the distance $\mathrm{x}$.

Measurement of the distance $\mathrm{x}$ is found by using the ruler. The experiment was performed with at least 10 balls for each liquid. Knowing the values of $\kappa, h, \rho, \rho_{0}$ and $\mathrm{t}$, the dynamic viscosity coefficient of the fluid is determined using the formula (1).

\section{- Measurement of the kinematic viscosity}

Capillary glass viscometer VPJ-4 (Fig. 5) is one of commonly used devices to measure the viscosity coefficient of liquids and it was designed to determine the kinematic viscosity of liquids in accordance with GOST 33-66. It's measuring range is from $0.6 \mathrm{~mm}^{2} / \mathrm{s}$ to $10000 \mathrm{~mm}^{2} / \mathrm{s}$ and it was very easy to measure and it is also a popular method to measure in laboratory conditions.

Capillary viscometer VPJ-4 (Fig. 5) is a device in the form of a U-shaped tube, the elbow (1) is soldered with the capillary (5). 
Measuring viscosity by a viscometer is based on the determination of the time, which a certain volume of a liquid will flow out through the capillary.
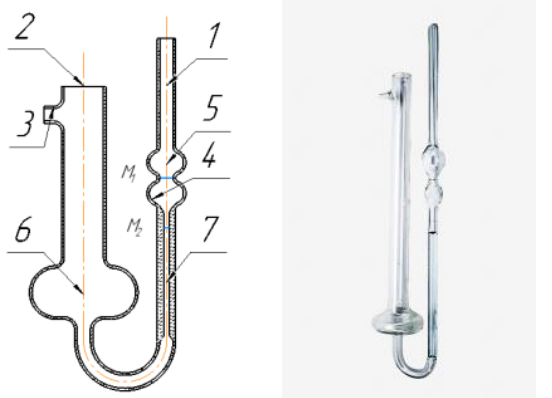

Figure 5: VPJ-4 capillary viscometer. 1,2 - bend of the viscometer; 3- discharge pipe; 4- an upper reservoir of the viscometer; 5 - capillary; 6 - bottom reservoir of

the viscometer; $M_{1}$ and $M_{2}$ - labels, limiting the measured volume of the lower reservoir viscometer

To determine the flow time to the discharge pipe (3), wear a rubber tube connected to a rubber bulb is inserted. Then, holding the bend (2), the viscometer is turned over and the bend (1) is immersed in a reservoir with liquid. The liquid is sucked in (with a pear) to the $M_{1}$ mark, while air bubbles should not form in the liquid. At the moment when the liquid reaches the $M_{1}$ mark, the device is removed from the vessel and immediately turned over to its original position. Remove excess fluid from the bend (1), remove the rubber tube from the bend (2), and put it on the bend (1). Then the viscometer is placed in the thermostat so that the expansion (4) is lower than the liquid level on the thermostat. It is kept in a thermostat for more than 30 minutes at a given temperature and then the liquid is sucked into the bend to the level of one-third of the expansion (4). Disconnect the rubber tube and measure with a stopwatch the time for the meniscus level to drop from the M1 mark to the M2 mark.

The diameter of the capillary is $3.55 \mathrm{~mm}$. The kinematic viscosity of the liquid was determined by using the following equation [GOST 33-66],

$$
V=\frac{g}{9.807} \times T \times K
$$

Where:

$\mathrm{K}$ - Constant of the viscometer $\left(9.224 \mathrm{~mm}^{2} / \mathrm{s}^{2}\right.$ according to GOST 33-66);

$\mathrm{V}$ - Kinematic viscosity of liquid $\left[\mathrm{mm}^{2} / \mathrm{s}\right]$;

$\mathrm{T}$ - Liquid flow time in seconds;

\section{Results and Discussions}

For analysing the influence of the magnetic and pulsated electromagnetic fields on the dynamic and kinematic viscosity coefficient of fluid, we magnetized three various cutting fluids in flowing conditions in 30 minutes. We used 8 permanent magnets with $100 \mathrm{mT}$ magnetic field strength for making a stationary magnetic field and we used three different magnetic field strengths such as $40 \mathrm{mT}, 60 \mathrm{mT}$ and $80 \mathrm{mT}$. The electromagnetic treatment equipment is prototype equipment of 12 layer solenoid coils. Each layer contains 175 turnsoff wires with a wire thickness of $1.06 \mathrm{~mm}$. The pipe of the equipment has an internal diameter (18.5 $\mathrm{mm}$ ) and external diameter $(25 \mathrm{~mm})$ with $6.5 \mathrm{~mm}$ of wall thickness. The length of the solenoid coil is 200 $\mathrm{mm}$ (Fig. 3). The core of the device is made of ceramic material. The device was fixed to produce maximum $80 \mathrm{mT} 50 \mathrm{~V} / 8 \mathrm{~A}$ with a power consumption of 400 watts/h.

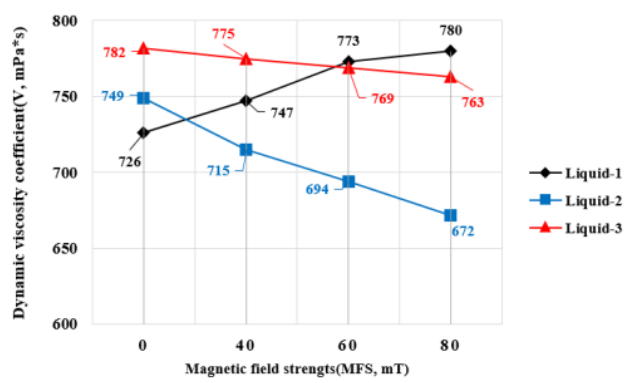

(a)

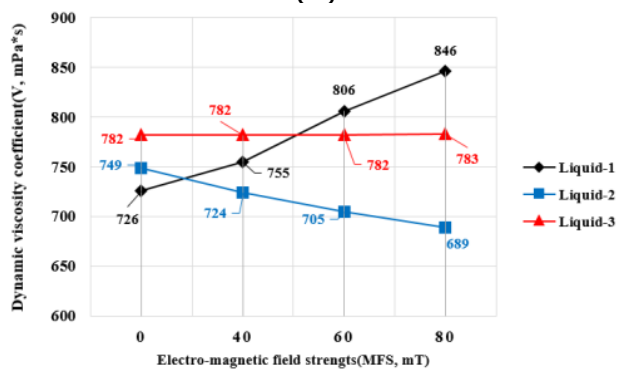

(b)

Figure 6: Influence of the stationary magnetic field (a) and electromagnetic field (b) on the dynamic viscosity coefficient of fluids in different magnetic field strengths

Line graphs given on the Fig. 6 (a) and (b) show the difference between the effect of magnetic and pulsed electromagnetic fields on the dynamic viscosity coefficient of cutting fluids.

The experiment, which was conducted in liquid-1 shows that a pulsed electromagnetic field affects it more than a stationary magnetic field. When the liquid-1 (tap water) was affected by the same magnetic and electromagnetic field strengths (80 $\mathrm{mT}$ ) the dynamic viscosity coefficient was $780 \mathrm{mPa}$ * and $846 \mathrm{mPa}^{*}$ s respectively.

The viscosity coefficient of not magnetized liquid1 was $726 \mathrm{mPa}^{*} \mathrm{~s}$ and under the effect of the electromagnetic field, it was increased by $14 \%$ while the stationary magnetic field caused $7 \%$ of increase on the viscosity coefficient of liquid-1.

However, magnetic and electromagnetic fields caused a decrease in liquid-2. While the dynamic viscosity coefficient of magnetized liquid-2 was 749 
$\mathrm{mPa} *$, it dropped at $672 \mathrm{mPa}$ s after the influence of the permanent magnetic field $(80 \mathrm{mT})$ and dropped at $689 \mathrm{mPa}^{*} \mathrm{~s}$ under the effect of the pulsed electromagnetic field $(80 \mathrm{mT})$.

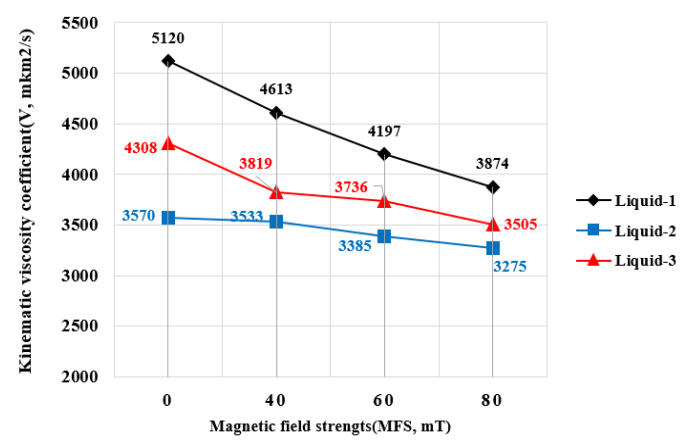

(a)

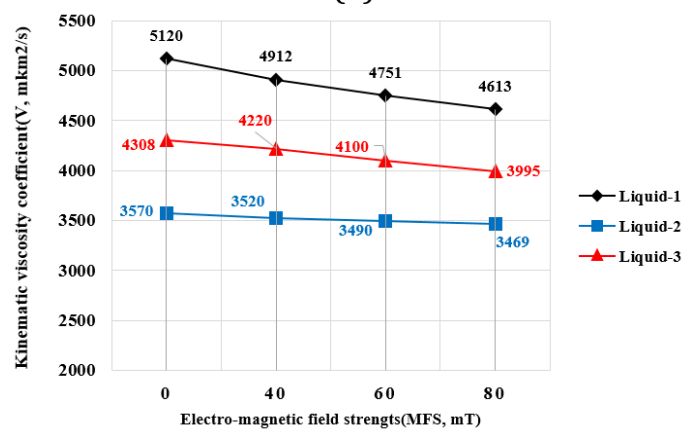

(b)

Figure 7: Influence of the stationary magnetic field (a) and electromagnetic field (b) on the kinematic viscosity coefficient of fluids in different magnetic field strengths

The results obtained from the third experiment differed from the previous two. The dynamic viscosity coefficient of liquid-3 was almost not influenced by pulsed electromagnetic field while the stationary magnetic field decreased the dynamic viscosity coefficient of not magnetized liquid-3 from $782 \mathrm{mPa}^{*}$ s to $763 \mathrm{mPa}^{*}$ s.

The difference between the influence of magnetic and pulsed electromagnetic fields on the kinematic viscosity coefficient of cutting fluids was also found in the experiment. Fig. 7 (a) and Fig. 7 (b) show that the magnetic field can cause to the decrease (15\%) on the kinematic viscosity coefficient of liquid-1 from $5120^{*} 10^{-3} \mathrm{~mm}^{2} / \mathrm{s}$ to $3840^{*} 10^{-3} \mathrm{~mm}^{2} / \mathrm{s}$ when the magnetic field strength was changed from $0 \mathrm{mT}$ to $80 \mathrm{mT}$. However, under 30 minutes of the effect of the electromagnetic field $(80 \mathrm{mT})$, it was dropped (10\%) until $4613^{*} 10^{-3} \mathrm{~mm}^{2} / \mathrm{s}$. Kinematic viscosity coefficient was also decreased in liquid-2 in the same order as it was in liquid-1.

The kinematic viscosity coefficient of liquid- 2 was $3570^{*} 10^{-3} \mathrm{~mm}^{2} / \mathrm{s}$, but it decreased until $3275^{*} 10^{-3}$ $\mathrm{mm}^{2} / \mathrm{s}$ after $80 \mathrm{mT}$ of magnetic treatment. However, the influence of the electromagnetic field $(80 \mathrm{mT})$ causes three percent of decrease (until $3469^{*} 10^{-3}$ $\mathrm{mm}^{2} / \mathrm{s}$ ) in kinematic viscosity coefficient. While the results reached from the liquid-3 have different numbers but the order is the same as other differences. Clearly, the kinematic viscosity of liquid3 was decreased from $4308^{*} 10^{-3} \mathrm{~mm}^{2} / \mathrm{s}$ to $3505^{*} 10^{-}$ $3 \mathrm{~mm}^{2} / \mathrm{s}$ after magnetic treatment $(80 \mathrm{mT})$, while it dropped until $3995^{*} 10^{-3} \mathrm{~mm}^{2} / \mathrm{s}$ under the effect of a pulsed electromagnetic field ( $80 \mathrm{mT})$.

Our experimental results show that there is a difference between the effect of magnetic and pulsed electromagnetic fields on the dynamic and kinematic viscosity coefficient of cutting fluids including water. Moreover, it was investigated that the effect of magnetic and electromagnetic fields on the dynamic viscosity coefficient of liquids depends on the content of liquids. Because of the results obtained from the experiment, liquids with a various content were influenced differently by the same magnetic and electromagnetic field strength.

The results obtained from the experiment show that the pulsed electromagnetic field affects the dynamic viscosity coefficient more than a stationary magnetic field. However, the magnetization effect on dissolved liquids (liquid-2 and liquid-3) is more in the stationary magnetic field than a pulsed electromagnetic field. Measurement of the kinematic viscosity coefficient of liquids also proves that a stationary magnetic field causes more changes in the physical properties of liquids than a pulsed electromagnetic field. It is essential to point that the magnetic field strength, magnetizing condition, and velocity of liquids were considered as three influential factors that affected the changes caused by the magnetic and electromagnetic field $[11,12]$.

Therefore, all of the experiments have been conducted in the same laboratory condition and at the same flowing velocity of the liquid $(v=0.4 \mathrm{~m} / \mathrm{s})$.

However, the method of magnetic field treatment is unclear, some researchers have done it to understand and clarify it. The theory was suggested by the authors wat that the hydrogen bonds among water molecules were likely affected by the magnetic field [13-18]. But still, there is not comprehensive information about the influence of magnetic field treatment.

\section{Conclusions}

Based on the results obtained from the experiment in this manuscript, the following conclusions can be drawn.

A stationary magnetic field affects the dynamic and kinematic viscosity coefficient of liquids more than a pulsed electromagnetic field. However, the influence of a pulsed electromagnetic field on the viscosity coefficient of fluids is higher than the influence of a magnetic field when the fluid has fewer dissolved solids. 
The influence of magnetic fields on liquids depends on dissolved elements. In the experiment, dissolved liquids (liquid-2 and liquid-3) have less change on the dynamic and kinematic viscosity coefficient than tap water (liquid-1) after magnetic (electromagnetic) treatment.

\section{References}

[1] K. Chang and C.; The effect of an external magnetic field on the structure of liquid water using molecular dynamics simulation, Journal of Applied Physics ,2006.

[2] Y.I. Cho, S. Lee.; Reduction of the surface tension of water due to physical water treatment for fouling control in heat exchangers. International Communication in Heat and Mass Transfer, Volume 32, Pp: 1-9, 2005.

[3] M.C. Amiri, A.A. Dadkhah.; On the reduction in the surface tension of water due to magnetic treatment. Colloids and Surfaces A: Physiochem. Eng. Aspects, Volume 278, Pp: 252-255, 2006.

[4] Z. Liu, X. Gao, J. Zhao, \& Y. Xiang.; The Sterilization Effect of Solenoid Magnetic Field Direction on Heterotrophic Bacteria in Circulating Cooling Water. Procedia Engineering, Volume 174, Pp: 1296-1302, $2017 . \quad$ doi: https://doi.org/10.1016/i.proeng.2017.01.274.

[5] C. Baresel, V. Schaller, C. Jonasson, C. Johansson, R. Bordes, V. Chauhan, A. Sugunan, J. Sommertune \& S. Welling; Functionalized magnetic particles for water treatment. Heliyon, Volume 5, Issue 8, 2019. doi: https://doi.org/10.1016/j.heliyon.2019.e02325.

[6] E.O. Umarov, U.T. Mardonov, U.K. Shoazimova; Influence of the Magnetic Field on the Viscosity Coefficient of Lubricoolant that is used in the Cutting Proces. International Journal of Mechatronics and Applied Mechanics, Volume 8, Issue 2, Pp: 144-149, 2020. doi: https://www.doi.org/10.17683/ijomam/issue8. $\underline{50 .}$

[7] E.0. Umarov, U.T. Mardonov, \& M.Z. Turonov; Measurement of dynamic viscosity coefficient of fluids, presented at the Euro-Asia Conferences, New York, USA, January 20, 2021.

[8] Y. Wang, B. Zhang, Z. Gong, et al; The effect of a static magnetic field on the hydrogen bonding in water using frictional experiments. J Mol Struct, Volume 1052, issue 11, 102-104, 2013. doi: https://doi.org/10.1016/j.molstruc. 2013.08.021.

[9] R. Cai, H. Yang, J He, et al.; The effects of magnetic fields on water molecular hydrogen bonds. J Mol Struct, Volume 938, 2009. doi: https://doi.org/10.1016/j.molstruc.2009.08.037.

[10] E.L. Toledo, T.C. Ramalho, Z.M. Magriotis; Influence of magnetic field on physical-chemical properties of the liquid water: insights from experimental and theoretical models. J Mol Struct, Volume 888, issues 1-3, Pp: 409-415, 2008. doi: https://doi.org/10.1016/j.molstruc.2008.01.010.
[11] B. Liu, B. Gao, X Xu; The combined use of magnetic field and iron-based complex in advanced treatment of pulp and paper wastewater. Chem Eng J, Volume 178, issue 1, Pp: 232-238, 2011 doi: https://doi.org/10.1016/j.cej.2 011.10.058.

[12] J.J. Manyà, M.A. Jr, C.K. Kinoshita, et al.; Specific heat capacity of pure water at $4.0 \mathrm{MPa}$ between 298.15 and 465.65 K. Ind Eng Chem Res, Volume 50, issue 10, Pp: 6470-6484, 2011 doi:https://doi.org/10.1021/ie102462g.

[13] Y. Wang, B. Zhang, Z. Gong, et al; The effect of a static magnetic field on the hydrogen bonding in water using frictional experiments. J Mol Struct, Volume 1052, issue 11, Pp: 102-104, 2013. doi: https://doi.org/10.1016/j.molstruc. 2013.08.021.

[14] R. Cai, H. Yang, J. He, et al; The effects of magnetic fields on water molecular hydrogen bonds. J Mol Struct, Volume 938, Issues 1-3, Pp: 15-9,

2009. doi:https://doi.org/10.1016/j.molstruc.2009.08. 037

[15] E.L. Toledo, T.C. Ramalho, Z.M. Magriotis; Influence of magnetic field on physical-chemical properties of the liquid water: insights from experimental andtheoretical models. J Mol Struct, Volumes 888, Issues 1-3, Pp: 409-415, 2008. doi: https://doi.org/10.1016/j.molstruc.2008.01.010

[16] T. Nodir, T. Sherzod, Z. Ruslan, T. Sarvar, \& B. Azamat. Studying the scientific and technological bases for the processing of dumping copper and aluminum slags. Journal of Critical Reviews, Volume 7, Issue 11, Pp: 441-444, 2020. doi: http://dx.doi.org/10.31838/jcr.07.05.95.

[17] Turakhodjaev, N., Tursunbaev, S., Tashbulatov, S., \& Kuchkorova, M.; Analysis of technological solutions for reducing the copper concentration in slags from oxygen-flare smelting of copper sulfide concentrates. Journal of Critical Reviews, Volume 7, Issue 5, Pp: 449-452, 2020.

[18] Erkin, U., Umidjon, M., \& Umida, S. (2021, September). Application of Magnetic Field on Lubricating Cooling Technological Condition in Metal Cutting Process. In International Conference on Reliable Systems Engineering (pp. 100-106). Springer, Cham.

[19] Nodir, T., Sarvar, T., Andrey, J., \& Yahyojon, M.; Mathematical Model for Calculating Heat Exchange. In International Conference on Reliable Systems Engineering, Springer, Cham, Pp: 243-249, 2021.

[20] Umarov, E., Mardonov, U., Abdirakhmonov, K., Eshkulov, A., \& Rakhmatov, B. (2021). Effect of magnetic field on the physical and chemical properties of flowing lubricating cooling liquids used in the manufacturing process. IIUM Engineering Journal, Volume 22, Issue 2, Pp: $327-$ 338, $2021 . \quad$ doi: https://doi.org/10.31436/iiumej.v22i2.1768. 